\title{
Die Grenzen der webbasierten Konsumentenmacht
}

\author{
Neue technische Möglichkeiten zur Information \\ und interaktiven Kommunikation werden oft als \\ neue Möglichkeiten zur Stärkung von Demokra- \\ tie und bürgerschaftlichem Engagement gedeu- \\ tet. Doch inwiefern bieten netzbasierte Formen \\ der Kommunikation tatsächlich neue Chancen \\ zur Förderung nachhaltigen Konsums? \\ Von Sigrid Baringhorst
}

N icht nur in Bezug auf die Stärkung der Demokratie, auch hinsichtlich der Erweiterung des Wissens über Konsum und die Entfaltung einer kollektiven Macht ethisch motivierter Konsumenten wird netzbasierten Kommunikationsmedien eine hohe Bedeutung zugeschrieben. Welche Pozentiale und Reichweiten haben diese netzbasierten Formen der Kommunikation hinsichtlich der Handlungsabstimmung kollektiver Aktionen zur Beeinflussung des Marktes oder zur Beeinflussung verbraucherpolitischer Normsetzungen? Wie schon in der Fragestellung angedeutet, verbietet sich ein generalisiertes Sprechen über die Politik im Netz ebenso wie über nachhaltigen Konsum im Netz. Kommunikationsangebote wie E-mail, Twitter, Weblogs, Videoportale, Corporate Watch Portale oder Social Networking Sites unterscheiden sich in Form und Ausmaß der Partizipationsmöglichkeiten; zudem unterscheiden sich die verschiedenen Angebote in ihrer inhaltlichen und normativen Ausrichtung. Während einige Kommunikationsangebote, wie die 2009 gegründete Plattform www.greenaction.de der GreenAction Community von Greenpeace, durch kollektiv gestützte öffentlichkeitswirksame Kampagnen auf allgemein politische wie unternehmenspolitische Anschlussreaktionen zielen, überschreiten viele andere Webangebote, wie etwa der Webblog von Kirsten Brodde www.kirstenbrodde.de, die engen Grenzen einer ökologisch und sozial verantwortlichen Lebensstilpolitik im Sinne einer bloßen LOHAS-Orientierung kaum (Yang et al. 2010).

\section{Soziale Netzwerke und Nachhaltigkeit}

Im Vorfeld eines Forschungsprojekts zu „Consumer Netizens. Neue Formen der Bürgerschaft an der Schnittstelle von politischem Konsum und Social Web“ wurde im Januar 2010 an der Universität Siegen eine Suchmaschinenrecherche durchgeführt, um aufbauend auf eigenen früheren Forschungsergeb- nissen konsumkritische und ökologiebewusste Social Web-Projekte zu identifizieren (Baringhorst et al. 2010). Dabei ergab die Recherche mit Suchwörtern wie „Boykott“ oder „Buykott“ allein im deutschen Sprachraum achtzig Beispiele. Systematisch lässt sich die Vielzahl der Social Web-Angebote zum nachhaltigen Konsum grob nach drei Typen unterscheiden. Erstens, individuelle Kommunikationsangebote wie die oben genannten Weblogs zu nachhaltigem Konsum; zweitens, mehr oder weniger politisierte Social Networking Sites wie zum Beispiel www.utopia.de, auf denen Mitglieder eigene Nutzerprofile entwickeln und sich untereinander mehr oder weniger moderiert austauschen und kollaboratives Konsumwissen generieren und bewerten, sowie drittens, kampagnenorientierte Kommunikationsformen wie sie Campact, Greenpeace oder andere Nichtregierungsorganisationen oder Netzwerkorganisationen wie Attac entwickelt haben. Die Trennung zwischen individueller und kollektiver Handlungsorientierung ist dabei eher eine analytische, da in vielen Fällen die Grenzen zwischen praktischer Konsumberatung und Gesellschaftskritik ebenso fließend sind wie die zwischen privater und öffentlicher Sphäre der Konsumkommunikation.

In der öffentlichen Berichterstattung über nachhaltigen Konsum im Netz haben in den letzten Jahren vor allem Carrot Mobs und die Social Networking Plattform der Utopisten viel Resonanz erfahren. Aber auch individuelle konsumkritische Netzaktionen können, wie ein oft zitiertes amerikanisches Fallbeispiel zeigt, beträchtliche öffentliche Wirkung entfalten. Im Folgenden sollen diese für die beiden erstgenannten Kommunikationsmodi illustrativen Beispiele kurz skizziert und bewertet und im Anschluss mit eher kampagnenartigen Formen der Netzkommunikation verglichen werden.

\section{Individuelle Netzaktionen: Der Fall Peretti}

Jonah Peretti, damals Assistant Adjunct Professor an der New York University, schrieb am 5. Januar 2001 eine E-Mail an den Sportartikelhersteller Nike in Reaktion auf das Angebot des Unternehmens, Designvorschläge von Verbrauchern umzusetzen (Peretti 2004). Er bestellte beim Unternehmen ein Paar Turnschuhe mit der provozierenden Aufschrift sweatshop und löste damit einen längeren E-Mail-Austausch mit dem OnlineVerbraucherservice des Unternehmens aus. Dieser wurde mit der spöttischen Aufforderung Perettis beendet: „Could you please send me a color snapshot of the ten-year-old Vietnamese girl who makes my shoes? “ In kurzer Zeit wurde im elektronischen Schneeballsystem die Kommunikation mit dem $\rightarrow$ 
Unternehmen millionenfach repliziert. Lokale, regionale bis hin zu internationalen Zeitungen griffen die Story auf. Am Ende lud sogar der Fernsehsender NBC den jungen Wissenschaftler ein, in seiner Today Show mit einem Vertreter des skandalisierten Unternehmens über soziale Unternehmensverantwortung zu sprechen.

\section{Carrot Mobs: Mobilisierung von Konsumenten auf lokaler Ebene}

Vor allem in europäischen und amerikanischen Großstädten werden seit 2008 unter dem Begriff „Carrrot Mobs“ bekannt gewordene „Flashmobs“ über das Internet organisiert. Dem Prinzip des „Buykotts“ folgend zielen Carrot Mobs auf die Förderung nachhaltigen Konsums, indem bestimmte ökologisch korrekte Geschäfte für klimaschonende Investitionsabsichten honoriert werden. Carrot Mob Aktionen, wie sie in Deutschland zuerst von Carrot Mob Berlin initiiert wurden, beginnen mit einer Anfrage nach der Bereitschaft von Geschäften, in einer bestimmten Sparte einen möglichst hohen Teil ihrer Tageseinnahmen für Maßnahmen der $\mathrm{CO}_{2}$ Reduzierung auszugeben (1). Nach der Auswahl des besten Investitionsangebots legen Carrot Mob Organisatoren und Geschäftsinhaber vertraglich die Einnahmeanteile fest, die für die angegebenen Zwecke genutzt werden sollen. Als Gegenleistung dazu versammelt sich im ausgewählten Geschäft an einem zuvor bestimmten Tag eine Gruppe nachhaltiger Konsumenten. Per Facebook und Twitter aber auch aufgrund von Radio- und Zeitungskontakten werden potenzielle Käufer und Interessierte auf die Aktion aufmerksam gemacht und zum Konsum aufgefordert. So kamen bei einer Kaufaktion in Köln im April 2010 nach WDR-Aussagen durch 350 zusätzliche Käufer insgesamt 2.937,50 Euro zusammen, die auf Basis einer professionellen Klimaberatung für klimaschonende Investitionen in dem dort ausgewählten Obst- und Gemüsegeschäft genutzt werden sollten. Hinsichtlich der Akteure scheinen eine hohe Fluktuation und eine Mobilisierung auf Basis nicht organisatorisch verfestigter Freundschaftsnetzwerke typisch zu sein. Manchmal gibt es aber auch Koalitionen mit organisierten Gruppen wie etwa der Berliner Jugend des BUND in einer Aktion des Carrot Mob Berlin.

\section{Utopia.de: An der Schnittstelle zwischen Unternehmen und Verbrauchern}

Betrachtet man die Zahl der registrierten User, so ist www.utopia.de, das im November 2007 gegründete Webportal für strategischen Konsum und nachhaltigen Lebensstil mit im August 2011 mehr als 60.000 Mitgliedern die erfolgreichste Social Web Plattform zur Förderung nachhaltigen Konsums in Deutschland. Nach dem Selbstverständnis der das Portal bereitstellenden Utopia AG ist Utopia „der Motor des Aufbruchs und für alle diejenigen - Konsumenten wie Unternehmen - der Ort, um sich zu sammeln, miteinander zu vernetzen und dann gemeinsam loszulegen, um mittels des strategischen Konsums den Markt in eine positive Richtung zu lenken. Kaufen und gönnen Sie sich was, aber das Richtige!“ (2) Registrierten Nutzern werden neben emotionalen, gemeinschaftsbildenden vor allem alltagsweltlich-praktische Nutzen gegeben. So gibt der von der Deutschen Bundesstiftung Umwelt geförderte Online-Einkaufsführer Utopia-City den Usern ein Geo-Medium, das auch mobil abrufbar, bundesweit gute Adressen offeriert: „Bio-Lebensmittel, Naturkosmetik oder Möbel aus nachhaltiger Herstellung, das nächste Café mit vegetarischen Gerichten, eine umweltfreundlich arbeitende Druckerei oder eine Vermietung für Elektrofahrzeuge - hier gibt es alles, was das Konsumentenherz begehrt.“ (3) Der Utopia Produkt Guide, gefördert durch das Umweltministerium des Landes Baden-Württemberg, nutzt kollaborativ erzeugtes Utopisten-Wissen zur Bereitstellung individuell abfragbarer Produktbewertungen von Öko-Energieanbietern beispielsweise zu korrekten Schwammtüchern (4).

In der alternativen Szene durchaus kritisch kommentiert ist die enge Verbindung, die die Utopia AG inzwischen mit zahlreichen mehr oder weniger ökologisch ausgerichteten Unternehmen eingegangen ist. Utopia versteht sich „als Schnittstelle zwischen der Industrie und den nachhaltigen Wünschen und Bedürfnissen der Verbraucher" und zielt neben dem Community-Building nachhaltiger Verbraucher vor allem auf den „Dialog mit Unternehmen“, die sie „in die Nachhaltigkeit begleiten“ will (5). In der hybriden Konstruktion der Utopia AG zwischen Non-Profit- und Profitorientierung verkörpert sich nicht nur die Schwierigkeit der Aufhebung des Widerspruchs zwischen basisnaher und zugleich professionell gestalteter Kommunikation. Damit verkörpert die Utopia-Plattform zugleich die widersprüchliche Geschichte der Netzkommunikation selbst, die einerseits von Beginn an vom Gedanken der Allmende und der Free Software Entwicklung getragen war, andererseits jedoch immer stärker werdenden kommerziellen Verwertungsinteressen unterworfen wurde.

\section{Grenzen gesellschaftlicher Wirksamkeit}

Die hohe Zahl registrierter Utopisten wie die beeindruckende Zahl der gleich nach der Einrichtung im Juli 2011 gezählten Aufrufe des von Bundesregierung und Verbraucherzentrale installierten neuen Webportals zu Lebensmittelklarheit, auf dem Verbraucher Produkte melden können, bei denen sie sich durch Aufmachung oder Kennzeichnung getäuscht fühlen, zeugt davon, dass interaktive konsumkritische Webangebote mit ihrer Kombination von Information, Beratung, Vernetzung, WatchDog und Beschwerdefunktion einen Nerv der Zeit getroffen haben (6). Die Opportunitätskosten des Engagements im Netz sind gering, die sozialen Bindungen in der Regel schwach und Aktionsbeiträge individuell steuerbar. Doch inwiefern ist der sogenannte strategische Konsum im Netz wirklich strategisch? Bedeutet die zunehmende Individualisierung und LifestyleOrientierung zugleich das Ende der Macht klassischer Intermediäre, das heißt vor allem zivilgesellschaftlicher Lobbygruppen? Und sind von zivilgesellschaftlichen Akteuren getragene kam- 
pagnenförmige Mobilisierungen durch die direkte Vernetzung von Verbrauchern im Netz sowie die direkte Kommunikation zwischen individuellen Verbrauchern und Regierungsinstitutionen oder Unternehmen obsolet geworden? Ich denke, nein. Denn das Netz wird nicht automatisch zur Netzöffentlichkeit. Um einer Privatisierung und Individualisierung der User entgegenzuwirken, bedarf es der Reintermediarisation der Netzkommunikation durch die reflexive und vermittelnde Tätigkeit zivilgesellschaftlicher Organisationen (Bohman 2004). Community-Plattformen wie utopia.de leisten dies angesichts der dominierenden Konsensorientierung, Entpolitisierung und Ausrichtung der Kommunikation an Bedürfnissen der individuellen Selbstdarstellung ebenso wenig hinreichend wie webbasierte regierungsoffizielle Beschwerdestellen. Aber auch Carrot Mobs lösen mit ihren sympathischen Buykott-Aktionen das strukturelle Problem der mangelnden Repräsentativität und der Bündelung der kaum zu überschauenden Vielzahl netzbasierter konsumkritischer Kommunikationsangebote nicht. Ihr Handlungsradius ist auf die lokale Ebene beschränkt, das mobilisierte Kaufkraftvolumen gering und ihre massenmediale Resonanz nicht zuletzt abhängig vom ihrem Neuigkeitswert.

Die Potenziale netzbasierter Konsumentenmobilisierung für die kollektive Erzeugung konsumkritischen Wissens wie für die produktive Vernetzung der vielen Einzelinitiativen zur Erzeugung markt- wie politisch wirksamer Verbrauchermacht in Online- wie in Offlineräumen sind noch keineswegs ausgeschöpft. Mehr netzbasierte Konsumentenmacht bedeutet jedoch auch mehr Anspruch an die demokratische Legitimität der Beteiligten. Doch worauf gründet sich die Macht konsumkritischer Meinungsführer im Netz? Inwiefern die interaktiven Plattformen kollaborativ erzeugter Beschwerdemacht selbst den zum Teil hoch gestellten Ansprüchen an Transparenz und Legitimität erfüllen, ist weiterhin kritisch zu beobachten.

\section{Anmerkungen}

(1) Im Internet unter: http://www.carrotmobberlin.com

(2) Quelle: http://www.utopia.de/ueber-utopia

(3) Der Online-Einkaufsführer Utopia-City im Internet: http://www.city.utopia.de

(4) Der Utopia Produkt Guide im Internet: http://www.utopia.de/produktguide/produkte

(5) Quelle: http://www.utopia.de/ueber-utopia

(6) Im Internet unter: http://www.lebensmittelklarheit.de/cps/rde/xchg/ lebensmittelklarheit

\section{Literatur}

Baringhorst, S. et al.: Unternehmenskritische Kampagnen im Zeichen digitaler Kommunikation. Wiesbaden 2010.

Bohman, J.: Expanding Dialogue: The Internet, Public Sphere, and Transnational Democracy. In: Shane, P. (Hrsg.): Democracy online. The Prospects for Political Renewal Through the Internet. London/New York 2004. S. 47-61.

Peretti, J. / Micheletti, M.: The Nike Sweatshop Email. Political Consumerism, Internet, and Culture Jamming. In: Micheletti, M. et al. (Hrsg.): Politics, Products, and Markets. Exploring Political Consumerism Past and Present. New Brunswick/London 2004. S. 127-142.

Yang, M. / Niesyto, J. / Baringhorst, S.: Politische Partizipation im Social Web. Das Beispiel nachhaltiger Konsumkritik. In: Wissenschaft und Umwelt Interdisziplinär, 14/2011. S.144-149.

\section{AUTORIN + KONTAKT}

Dr. Sigrid Baringhorst ist Professorin für Politikwissenschaft an der Universität Siegen.

Prof. Dr. Sigrid Baringhorst, Universität Siegen, Philosophische Fakultät, Seminar für Sozialwissenschaften, 57068 Siegen.

Tel.: +49 271740-4510/-4384; Fax: -2890;

E-Mail: Baringhorst@politikwissenschaft.uni-siegen.de

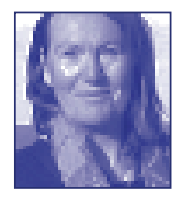

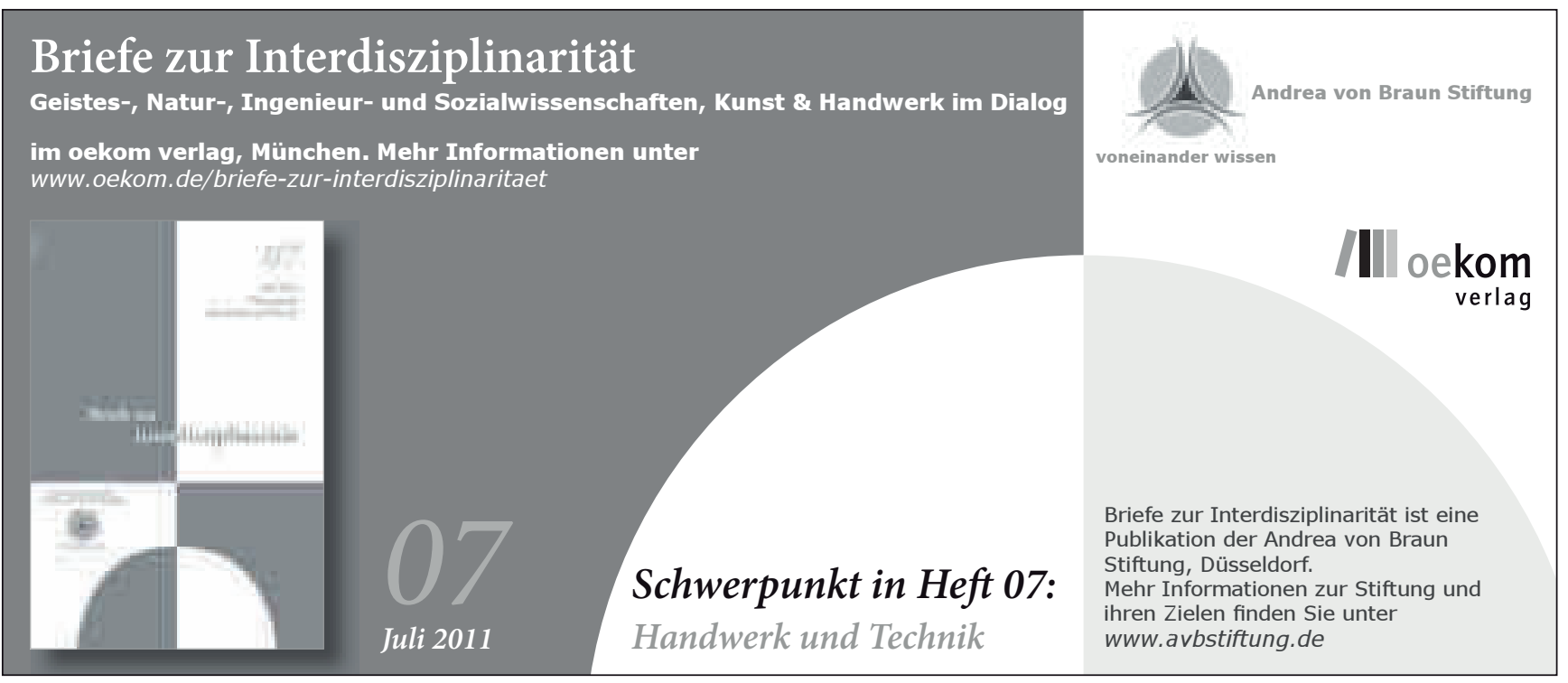




\section{Lizenzhinweis}

Die Beiträge in ÖkologischesWirtschaften werden unter der Creative-Commons-Lizenz "CC 4.0 Attribution Non-Commercial No Derivatives" veröffentlicht. Im Rahmen dieser Lizenz muss der Autor/Urheber stets genannt werden, das Werk darf nicht bearbeitet, abgewandelt oder in anderer Weise verändert und außerdem nicht kommerziell genutzt werden. Die digitale Version des Artikels bleibt für zwei Jahre Abonnent/innen vorbehalten und ist danach im Open Access verfügbar. 